\title{
Plasmid-based target protectors allow specific blockade of miRNA silencing activity in mammalian developmental systems
}

\author{
Jennifer L. Knauss ${ }^{1,2}$, Shan Bian' and Tao Sun ${ }^{1,2 *}$ \\ 1 Department of Cell and Developmental Biology, Weill Medical College of Cornell University, New York, NY, USA \\ ${ }^{2}$ Weill Cornell Graduate School of Medical Sciences, New York, NY, USA
}

\section{Edited by:}

Laure Bally-Cuif, Centre National de la Recherche Scientifique, France

Reviewed by:

Fen-Biao Gao, University of

Massachusetts Medical School, USA

\section{${ }^{*}$ Correspondence:}

Tao Sun, Department of Cell and Developmental Biology, Weill Medical College of Cornell University, 1300 York Avenue, Box 60, New York, NY 10065, USA

e-mail: tas2009@med.cornell.edu
Over the past decade, microRNAs (miRNAs) have emerged as essential posttranscriptional regulators of gene expression. Though a great deal has been discovered about miRNA genomics, biogenesis, mechanisms, and functions, the challenge of attributing phenotypes of altered miRNA expression to specific targets still remains. Here, we apply the existing target protector concept of blocking miRNA action at a single binding site in the $3^{\prime}$ untranslated region ( $3^{\prime} \cup T R$ ) of its target to a plasmid-based approach. We optimize and demonstrate target protector efficacy in vitro, where it blocks repression of a luciferase construct and an endogenous protein. Using the developing mouse cortex as a model, we validate that target protectors are effective in vivo, where protectors for the miR-19a binding sites in the Pten $3^{\prime}$ UTR alter proliferation and specification of neural progenitors, phenocopying Pten ectopic expression phenotypes. Our study introduces a new tool for analyzing specific miRNA:target interactions across mammalian developmental systems, facilitating further miRNA functional discoveries.

Keywords: miRNA, miR-19, Pten, mRNA protector, neural development

\section{INTRODUCTION}

MicroRNAs (miRNAs) are an extensive class of small non-coding RNAs that are critically important throughout development (Carrington and Ambros, 2003; Bartel, 2004; Alvarez-Garcia and Miska, 2005; Kloosterman and Plasterk, 2006). They regulate gene expression posttranscriptionally through incorporation into a RNA-induced silencing complex (RISC), which uses partial sequence complementarity to bind target messenger RNAs (mRNAs), usually in the $3^{\prime}$ untranslated region (3'UTR; Lewis et al., 2003). RISC binding can lead to blocking translation or enhancing the decay of target mRNAs, the final result being downregulation of the target protein (Bartel, 2004). Hundreds of miRNAs have been identified in the genomes of mammals including rodents and humans, and each miRNA can regulate many mRNAs in a cell-type specific manner, leading to a complex network of interactions (Landgraf et al., 2007). It is estimated that between 25 and $60 \%$ of human transcripts are regulated by miRNAs (Lewis et al., 2005; Lim et al., 2005; Friedman et al., 2009).

Conditional knockout and knockdown techniques have been used to examine the roles of miRNAs; however, there can be difficulty in interpreting molecular mechanisms underlying the observed phenotypes due to the large number of potential targets of each miRNA. Groups working in model systems such as zebrafish and Xenopus have bypassed this obstacle through a morpholino target protector approach, using sequence complementarity to block a miRNA from binding to a specific site (Choi et al., 2007; Bonev et al., 2011; Stanton and Giraldez, 2011). However, morpholinos are not a tractable tool in mammals and their short length of activity limits their application in developmental systems.
We here have developed a plasmid-based target protector system to tease apart the physiological roles of miRNAs in mammalian systems. Previous work in our lab has shown that in the developing cortex, miR-19a targets Pten mRNA (Bian et al., 2013). In the developing mouse cortex, Pten functions to repress progenitor expansion; therefore its repression by miR-19a results in increased proliferation (Groszer et al., 2001; Zheng et al., 2008). Thus, the miR-19a:Pten relationship provides an ideal readout for testing Pten derepression through target protectors. Here, we have designed and optimized target protectors for the miR-19a binding sites in the Pten $3^{\prime}$ UTR. We demonstrate that these target protectors can be electroporated in utero to allow functional investigation of a specific miRNA:mRNA interaction during cortical development in vivo. Our results provide a useful tool for investigation of long term, specific miRNA-target interactions both in vitro and in vivo using a plasmid-based target protector system.

\section{MATERIALS AND METHODS TARGET PROTECTOR DESIGN}

Protectors were designed as perfectly complementary sequence covering the miR-19a binding sites in the Pten $3^{\prime}$ UTR. The miRNA seed binding sequence was centered in the target protector, with complementary sequence on each side. Outside of the complementary sequence, restriction sites can be added as necessary for a cloning strategy.

For the second miR-19a binding site, target protectors with three lengths of complementarity to the Pten $3^{\prime}$ UTR were designed: 22,40 , and 60 nucleotides (nt; Figure 2A). All of the target protectors were designed to be the same total length as the $60 \mathrm{nt}$ protector and included junk sequences to increase their length as necessary, 
keeping the target protector in the middle of the construct. We ordered the target protectors as complementary oligonucleotides. After annealing, protectors were subcloned and inserted into the pCAGIG vector for electroporation and pCDNA3.1 for the luciferase assay.

\section{miR-19a EXPRESSION CONSTRUCT}

The precursor hairpin sequence of miR-19a and $\sim 100 \mathrm{nt}$ of genomic sequence flanking each side of the hairpin sequence was amplified by PCR from the genomic locus of the mouse miR-17-92 cluster. Sequences of primers are as following: miR-19a: F: 5'-CAGCTCGAGCAATCCAAGTCA-3', R: 5'-GCAGGCTCTACATCGACAC-3'. To generate the miR-19a expression construct, the miRNA fragment was inserted into pcDNA3.1 for transfection in cell lines, and pCAGIG for electroporation.

\section{LUCIFERASE ASSAY}

pGL4.13 firefly luciferase (Promega) vector was used for making constructs containing amplified 3'UTRs of targets. pGL4.73 renilla luciferase (Promega) was used as a transfection control. Plasmid DNA was quantified by UV spectrophotometry and used for transfection in a 6:2:1 ratio (protector:miRNA:target luciferase constructs) in Neuro2a (N2a) cells using Lipofectamine 2000 (Invitrogen) according to the manufacturer's protocol. Luciferase was activated using the Dual-Luciferase Reporter Assay kit (Promega) using the manufacturer's protocol and read on a Victor3 1420 multilabel counter (Perkin Elmer). Results were shown as firefly luciferase activity normalized to renilla as controls.

To make the $3^{\prime}$ UTR construct for the luciferase assay, a cDNA fragment encoding the mouse Pten $3^{\prime}$ UTR was amplified and subcloned into the pGL4.13 luciferase vector. The first miR19a binding site was mutated using QuikChange II Site-Directed Mutagenesis Kit (Agilent Technologies) according to manufacturer's instructions. All the primers for cloning of targets in the $3^{\prime} \mathrm{UTR}$ and their mutation are listed as the following: Pten3'UTR: F: 5'-CATCTAGAATACATCCACAGGGTTTTGACA-3', R: 5'-TTGAAGCCCTAATCCCAACTCT-3'; Pten-3'UTR-miR19a-mut1: $\quad 5^{\prime}$-CCGGGTTCACGTCCTACCCCATTACAATTGT GGCAACAGATAAGTTT-3'.

\section{NORTHERN BLOT ANALYSIS}

Total RNA was isolated from N2a cells transfected with either the $60 \mathrm{nt}$ target protector or the pcDNA3.1 empty vector using Trizol reagent (Invitrogen) according to manufacturer's instructions. RNA samples and 0.1-2 kb RNA ladder (Invitrogen) were denatured at $70^{\circ} \mathrm{C}$ for $15 \mathrm{~min}$ and cooled on ice. Ethidium bromide was added to the RNA ladder for visualization. The DNA control sample was denatured at $95^{\circ} \mathrm{C}$ and cooled on ice. Samples were loaded onto a $1 \%$ formaldehyde agarose gel and separated at room temperature. After running, the ladder band locations were marked on the gel.

Samples were transferred onto a nitrocellulose membrane using a semi-dry transfer method overnight. After transfer, the ladder band locations were marked on the membrane. After crosslinking for $4 \mathrm{~h}$ at $80^{\circ} \mathrm{C}$, the membrane was hybridized at $50^{\circ} \mathrm{C}$ overnight using a denatured DNA probe for the $60 \mathrm{nt}$ target protector. The probe was body-labeled with digoxygenin (DIG)-labeled nucleotides using the DIG DNA Labeling Kit (Roche), following manufacturer instructions. After washing, the RNA was detected using the CDP-star chemiluminescent substrate (Roche).

\section{WESTERN BLOT ANALYSIS}

Expression levels of Pten were analyzed by the Western blot analysis. Protein extracts were harvested by lysing N2a cells transfected with combinations miR-19a, 60 nt target protector, and empty vector with RIPA lysis buffer $\left(150 \mathrm{mM} \mathrm{NaCl}, 1 \mathrm{mM} \mathrm{Na}{ }_{4} \mathrm{P}_{2} \mathrm{O}_{7}\right.$, $1 \mathrm{mM} \mathrm{NaF}, 1 \mathrm{mM}$ EDTA, $1 \mathrm{mM}$ PMSF, $2 \mathrm{mM} \mathrm{Na}_{3} \mathrm{VO}_{4}$, 1\% NP40, $50 \mathrm{mM}$ Tris, $\mathrm{pH}$ 7.5) with complete ${ }^{\mathrm{TM}}$ EDTA-free protease inhibitor mixture (Roche Diagnostics, Indianapolis, IN, USA). The protein samples were boiled in SDS sample buffer for $10 \mathrm{~min}$ before loading onto $10 \%$ Tris-glycine gels as $10 \mu \mathrm{g}$ for each lane and transferred onto PVDF membrane (Pall Corporation, Pensacola, FL, USA). For immunoblotting, membranes were blocked with $5 \%(\mathrm{w} / \mathrm{v})$ non-fat milk powder in $0.05 \%$ TBST $[50 \mathrm{mM}$ Tris-Cl, pH 7.5, $150 \mathrm{mM} \mathrm{NaCl}$, with $0.05 \%$ (v/v) Tween-20] and incubated at $4^{\circ} \mathrm{C}$ overnight with the following primary antibodies which were diluted in $0.05 \%$ TBST with $5 \%$ non-fat milk: Pten and actin. After washing with TBST, membranes were incubated with specific HRP-conjugated secondary antibodies for $1 \mathrm{~h}$ at room temperature followed with extended washes with TBST. Immunoblot reactions were visualized using chemiluminescent substrate (Pierce, Rockford, IL, USA) on Kodak BioMax light films (Kodak, Rochester, NY, USA). The intensities of the bands were densitometrically quantified with the image software ImageJ.

The following antibodies were used: anti-Pten (rabbit, 1:1,000; Cell Signaling) and anti-Actin (rabbit, 1:400; Sigma).

\section{IN UTERO ELECTROPORATION}

In utero electroporation was performed as described by Saito (2006). Briefly, electroporation was conducted at E13.5 and the brain tissues were harvested $24 \mathrm{~h}$ later at E14.5. Plasmid DNA was prepared using the EndoFree plasmid maxi kit (Qiagen) according to manufacturer's instructions, and diluted to $2.5 \mu \mathrm{g} / \mu \mathrm{l}$. DNA solution was injected into the lateral ventricle of the cerebral cortex, and electroporated with five $50-\mathrm{ms}$ pulses at $35 \mathrm{~V}$ using an ECM830 electrosquareporator (BTX).

Wild type CD-1 mice were used for all experiments. For staging of embryos, midday of the day of vaginal-plug formation was considered E0.5; the first $24 \mathrm{~h}$ after birth were defined as P0. Animal use was overseen by the Animal Facility at Weill Cornell Medical College.

\section{TISSUE PREPARATION AND IMMUNOHISTOCHEMISTRY}

Mouse brains were fixed in 4\% paraformaldehyde (PFA) in phosphate-buffered saline (PBS) over night, incubated in 25$30 \%$ sucrose in PBS, embedded in OCT and stored at $-80^{\circ} \mathrm{C}$ until use. Brains were sectioned $(10-14 \mu \mathrm{m})$ using a cryostat. For antigen recovery, sections were incubated in heated $\left(95-100^{\circ} \mathrm{C}\right)$ antigen recovery solution ( $1 \mathrm{mM}$ EDTA, $5 \mathrm{mM}$ Tris, $\mathrm{pH} 8.0$ ) for 15-20 $\mathrm{min}$, and cooled down for 20-30 min. Before applying antibodies, sections were blocked in 10\% normal goat serum (NGS) in PBS with $0.1 \%$ Tween-20 (PBT) for $1 \mathrm{~h}$. Sections were 
incubated with primary antibodies at $4^{\circ} \mathrm{C}$ overnight and visualized using goat anti-rabbit IgG-Alexa-Fluor-488 and/or goat anti-mouse IgG-Alexa-Fluor-546 (1:350, Molecular Probes) for $1.5 \mathrm{~h}$ at room temperature. Images were captured using a Leica digital camera under a Zeiss confocal microscope.

Primary antibodies against the following antigens were used: bromodeoxyuridine (BrdU; 1:50, DSHB), Ki67 (1:500, Abcam), Pax6 (1:200, Covance, rabbit), Tbr2 (1:500, Abcam), GFP (1:1000, Abcam, chicken), and GFP (1:1000, Rockland, rabbit).

\section{CELL COUNTING}

Coronal sections were collected in the medial cortical region (at levels between the anterior commissure and the anterior hippocampus). At least four sections from each brain and three brains from different litters were chosen for antibody labeling. Positive cells were quantified in fixed areas of $318 \mu \mathrm{m} \times 318 \mu \mathrm{m}$ and normalized to the averaged empty vector control value.

\section{STATISTICS}

For the luciferase and Western blot assays, three independent experiments were performed. For electroporated mouse sections, at least three brains from each group were analyzed. Statistical comparison was made by an analysis of variance (unpaired Student's $t$-test).

\section{RESULTS}

\section{TARGET PROTECTOR DESIGN AND IN VITRO TESTING}

In a typical cell, miRNAs target multiple mRNAs through partial sequence complementarity (Figure 1A). The concept behind target protectors is that a construct with perfect complementarity to a specific miRNA-binding site will outcompete the miRNA for binding at that site (Figure 1B). Thus, a single miRNA target is derepressed while the others remain regulated, allowing analysis of the effects of a single miRNA:mRNA relationship. To apply this concept in mammalian development, we designed plasmid-based mRNA target protectors using miR-19a:Pten regulation, since our previous work and others have demonstrated the targeting effect of miR-19a on Pten (Mu et al., 2009; Olive et al., 2009; Mavrakis et al., 2010; Bian et al., 2013).

To optimize the minimum length of sequence complementarity necessary for maximum protector efficacy, we designed target protectors with three lengths of complementarity for the second miR-19a binding site in the Pten $3^{\prime}$ UTR (Figure 2A). We chose the shortest length of $22 \mathrm{nt}$ because this is the approximate size of most miRNAs (Figure 2A). We also designed protectors of 40 and $60 \mathrm{nt}$ to increase complementarity, binding strength, and specificity (Figure 2A). Our basic rules for protector design are: (1) each protector is centered over the predicted miRNA seed binding site, (2) the protectors have perfect complementarity along the length of the $3^{\prime} \mathrm{UTR}$, and (3) the protector must not overlap any other known functional sites in the $3^{\prime} U T R$. For the 22 and 40 nt target protectors, junk DNA sequences were inserted outside of the complementary region so that the overall length of all three constructs was constant with the $60 \mathrm{nt}$ protector.

We hypothesized that an effective target protector would block miR-19a activity in the $3^{\prime}$ UTR of Pten in a luciferase assay, resulting

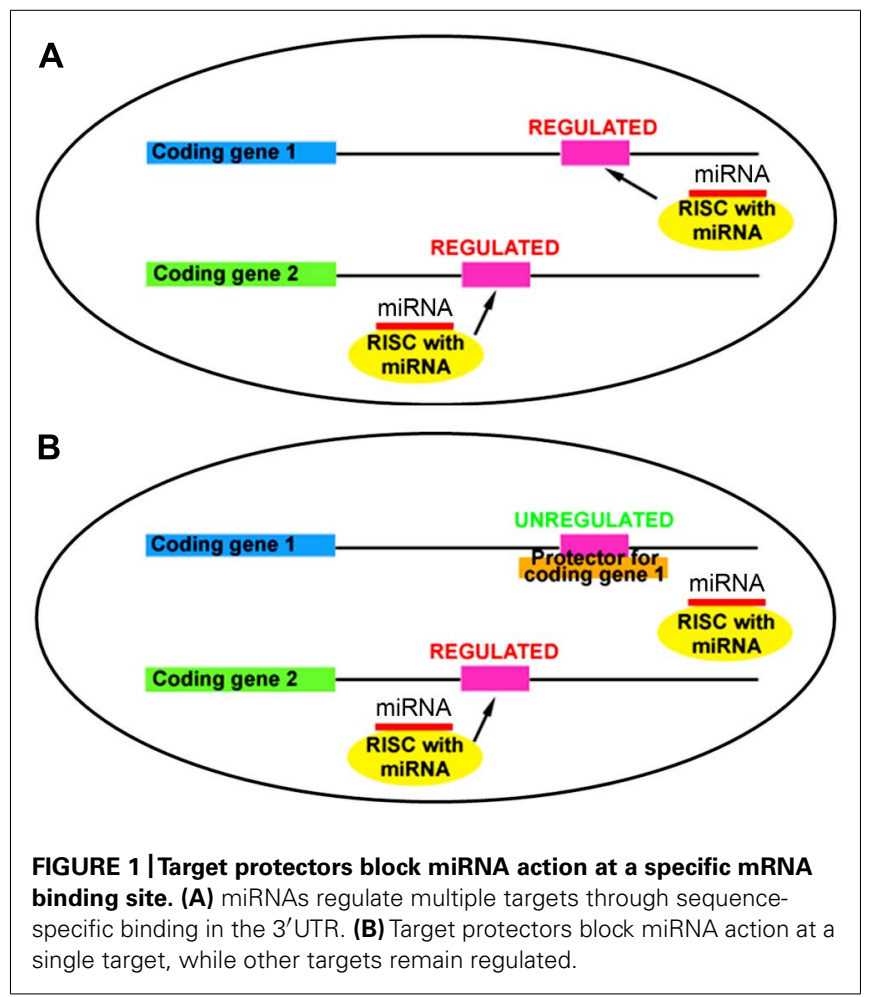

in a recovery of luciferase activity. To test the target protectors, we used a luciferase vector containing only the second miR-19a binding site of the Pten $3^{\prime}$ UTR (the first miR-19a binding site was mutated in the Pten $3^{\prime}$ UTR), and cotransfected it with miR19a and each target protector in N2a cells. In the absence of any target protector, luciferase activity of the Pten $3^{\prime}$ UTR was significantly decreased (Figure 2B). The 22 and 40 nt protectors did not show any significant recovery of luciferase activity, while the $60 \mathrm{nt}$ protector showed a significant, almost complete recovery of activity (Figure 2B). Neither miR-19a nor any of the target protectors had an effect on the luciferase activity of the Pten 3'UTR containing a mutation in both miR-19a binding sites (Figure 2B). Our results indicate that the $60 \mathrm{nt}$ target protector is the most effective at blocking miR-19a activity at the Pten $3^{\prime} \mathrm{UTR}$

\section{0 nt TARGET PROTECTOR BLOCKS miR-19a ACTIVITY IN VITRO}

To ensure that the $60 \mathrm{nt}$ target protector was transcribed and expressed as expected, we performed a northern blot assay using RNA extracted from N2a cells transfected with either the target protector or an empty vector. As a positive control, we also included digested vector DNA containing the protector. Based on the insert size and the predicted transcription start site and polyadenylation signals of the vector, the expect RNA size to be about 305 nt (Figure 3A). The protector is detected at the expected size in target protector transfected RNA and is not detected in the empty vector-transfected RNA, indicating that the protector is transcribed and expressed (Figure 3B).

We previously established that miR-19a regulates Pten posttranscriptionally by preventing its translation (Bian et al., 2013). Thus, we hypothesized that transfection of the protector should result in 


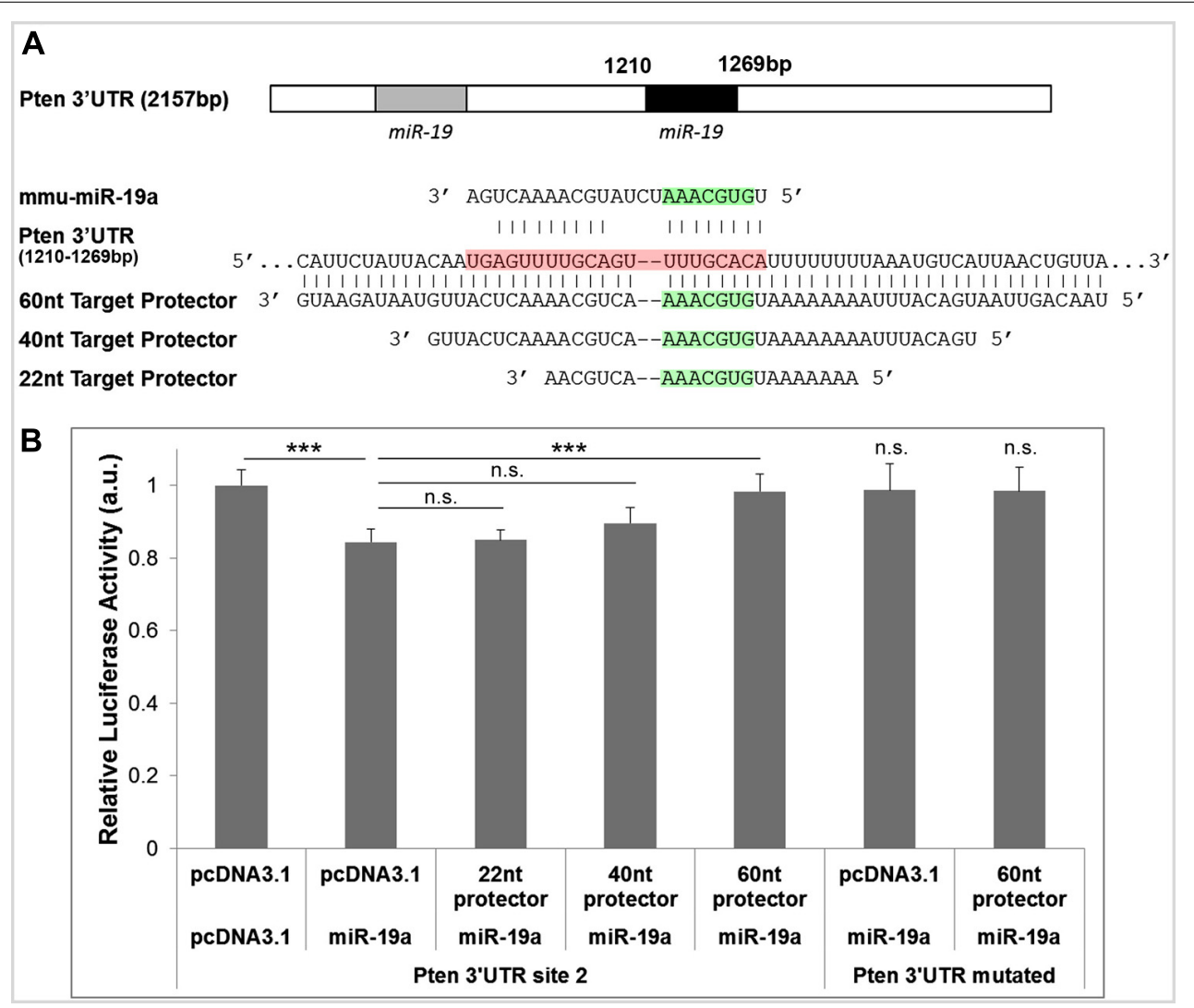

FIGURE 2 | Target protectors for Pten block miR-19a-induced repression. (A) Binding sites of miR-19a in the Pten $3^{\prime}$ UTR and complementary target protector sequences for the second miR-19a binding site. The seed binding sequence of miR-19a is highlighted in green, and the entire length of miR-19a along the Pten $3^{\prime}$ UTR is highlighted in red. (B) Luciferase assays of target protector effects on miR-19a repression of the second miR-19a binding site in the Pten $3^{\prime}$ UTR. miR-19a reduced luciferase activity in the absence of target protector. The $60 \mathrm{nt}$ target protector but not the 22 or $40 \mathrm{nt}$ target protector recovered luciferase activity of the Pten $3^{\prime}$ UTR. Neither miR-19a nor the $60 \mathrm{nt}$ target protector had an effect on the luciferase activity of the full length Pten 3'UTR when the miR-19a binding sites were mutated. Data are presented as mean $\pm \mathrm{SEM} ; n=3$ luciferase assays; $p$ values in relation to control $\left({ }^{* *} p<0.01\right)$. n.s., not significant. increased endogenous Pten protein in N2a cells. Indeed, a Western blot assay showed that transfection of only exogenous miR-19a results in a decrease of endogenous Pten, while cotransfection of exogenous miR-19a and the target protector, or the protector alone significantly rescues the endogenous Pten protein levels (Figures 3C,D). Our results suggest that the plasmid-based target protectors are transcribed and work to block posttranscriptional regulation by miRNAs.

\section{0 nt TARGET PROTECTOR BLOCKS miR-19a ACTIVITY IN VIVO}

Having established that the plasmid-based target protector is effective in vitro, we sought to apply it in vivo, where such a tool can provide insight to the function of specific miRNA-mRNA interactions during development. We previously established that miR-19a targeting of Pten promotes progenitor cell expansion in the developing mouse cortex (Bian et al., 2013). We expected that blocking miR-19a activity with the target protector will result in decreased proliferation of progenitors. To observe the maximum effect of blocking miR-19a binding to Pten, we also designed a $60 \mathrm{nt}$ target protector for the first binding site in the Pten $3^{\prime}$ UTR and used in utero electroporation to introduce protectors for both miR-19a binding sites in the Pten $3^{\prime}$ UTR into the embryonic day 13.5 (E13.5) cortex, analyzed at E14.5. The numbers of $\mathrm{BrdU}^{+}$and $\mathrm{Ki}^{+} 7^{+}$cells were significantly decreased after Pten target protectors were electroporated compared to empty vector electroporation, suggesting a functional result of the blockade of miR-19a silencing effect on Pten expression (Figure 4).

To test further whether decreased proliferation of neural progenitors in target protector electroporation is caused by upregulation of Pten, which is usually suppressed by miR-19a, we electroporated full length Pten containing the $3^{\prime}$ UTR with miR-19a binding sites (Pten-FL-3'UTR). We observed a significant decrease in both $\mathrm{BrdU}^{+}$and $\mathrm{Ki}^{+} 7^{+}$cells upon electroporation of the Pten overexpression construct compared to empty vector electroporation (Figures 4B,D). When Pten-FL-3' UTR was co-electroporated with exogenous miR-19a, the numbers of $\mathrm{BrdU}^{+}$and $\mathrm{Ki}^{+} 7^{+}$cells were recovered to wild type levels, indicating that the change is dependent on miR-19a activity on the Pten $3^{\prime}$ UTR (Figure 4). Our results demonstrate that plasmid-based target protectors can be electroporated into developing cortices to block miRNA action at a specific target. 

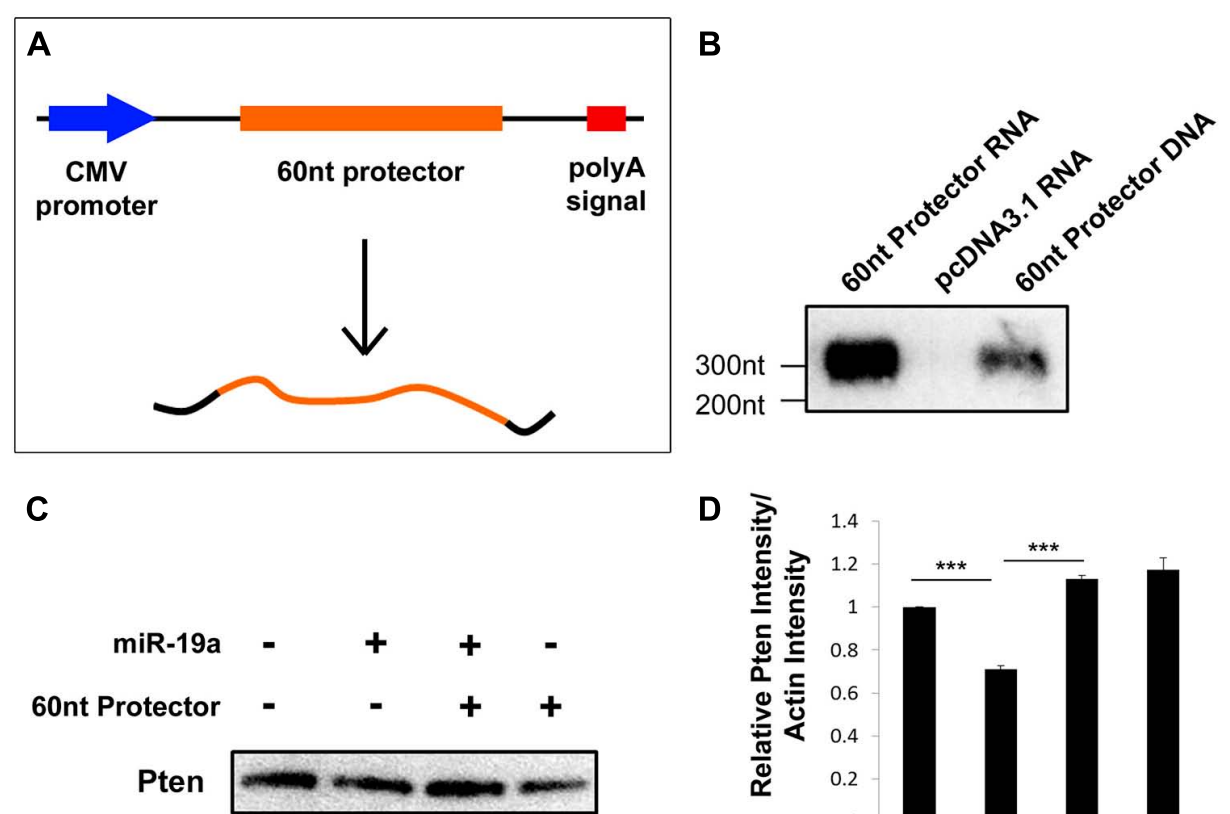

Actin

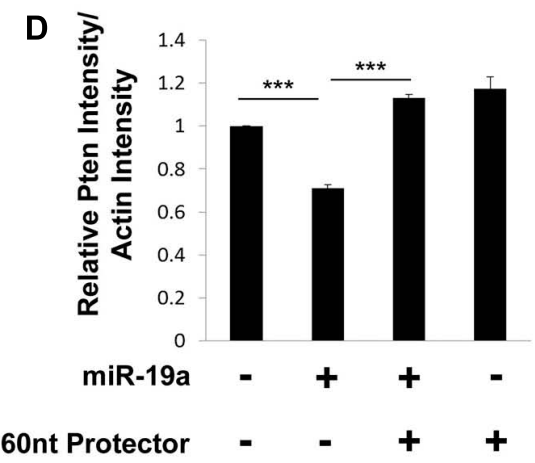

FIGURE 3 | Target protectors are transcribed and effective in vitro. (A) Schematic vector map of the $60 \mathrm{nt}$ target protector in pcDNA3.1. (B) The $60 \mathrm{nt}$ target protector and control target protector DNA were detected by Northern blot assays at the expected size. No target protector was detected in RNA extracted from empty vector-transfected cells. (C,D) The endogenous protein levels of Pten were decreased upon transfection of miR-19a but recovered upon cotransfection of miR-19a and the $60 \mathrm{nt}$ target protector. Transfection of the $60 \mathrm{nt}$ target protector alone also showed an increase in Pten. Data are presented as mean $\pm \mathrm{SD} ; n=3$ separate protein extractions; $p$ values in relation to control ( $\left.{ }^{* *} p<0.001\right)$.

\section{TARGET PROTECTORS REVEAL THE ROLE OF miR-19a REPRESSION OF Pten IN THE DEVELOPING CORTEX}

During corticogenesis, the transition of proliferative radial glia cells (RGCs) and intermediate progenitor (IP) into postmitotic neurons is tightly regulated in order to generate an appropriate amount of neurons while maintaining a progenitor pool. Our previous work has shown that the miRNA family miR-17-92, containing miR-19a, promotes RGC proliferation (Bian et al., 2013). To validate that miR-19a targeting of Pten is responsible for RGC proliferation, we introduced Pten target protectors into E13.5 cortices, which should result in a decrease of RGCs. We found that the number of Pax6 $6^{+}$RGCs is significantly decreased, while the number of Tbr2 ${ }^{+}$IPs is not changed (Figure 5).

To confirm further that Pten is responsible for the decrease of RGCs, we electroporated Pten-FL-3'UTR, which we expected to show a similar phenotype as the Pten target protectors, in E13.5 cortices collected at E14.5. Indeed, the number Pax6 ${ }^{+}$RGCs was decreased and the number of Tbr2 ${ }^{+}$IPs was not changed (Figures 5B,D). When Pten-FL-3'UTR and miR-19a were coelectroporated, the number of $\mathrm{Pax}^{+}$RGCs was recovered, while the number of Tbr2 ${ }^{+}$IPs remained unchanged (Figure 5). These results show that miR-19a targeting of Pten is critical for proliferation of RGCs in the developing cortex but does not affect the IP cell population. Using our Pten target protectors, we further demonstrate that the specific effects of miR-19a on RGC expansion occur through silencing Pten.

\section{DISCUSSION}

miRNAs have been proven to play critical roles in development of invertebrates and vertebrates (Carrington and Ambros, 2003; Alvarez-Garcia and Miska, 2005; Kloosterman and Plasterk, 2006). Since one miRNA has multiple targets, it has been a daunting task to demonstrate which genes are major targets in specific cells or tissues during development. In this study, we have designed a plasmid-based tool for analyzing specific miRNA:target relationships, shown that it works effectively in vitro and in vivo, and applied it to a miRNA:mRNA pair in the developing mouse cortex.

In the past, many studies have examined the roles of individual miRNAs by removing them globally or conditionally. In Drosophila and mice, genetic mutants have been generated, while in other systems such as zebrafish or Xenopus, morpholinos or other antisense oligonucleotides have been used to knockdown miRNAs (Sokol, 2005; Karres et al., 2007; Ventura et al., 2008; Woltering and Durston, 2008; Conte et al., 2010; Rasmussen et al., 2010). miRNA sponges with multiple miRNA-binding sites have also been used to soak up mature miRNAs (Ebert et al., 2007; Loya et al., 2009; Ebert and Sharp, 2010). While these methods provide a genetic approach to explore the overall function of a miRNA, they lack the power to attribute any phenotype to a particular mRNA target. Later, morpholino-based target protectors were applied in zebrafish and Xenopus to examine the importance of miRNA repression of a specific target (Choi et al., 2007; Bonev et al., 2011; Stanton and Giraldez, 2011). While this technique has been useful 
A PCAGIG

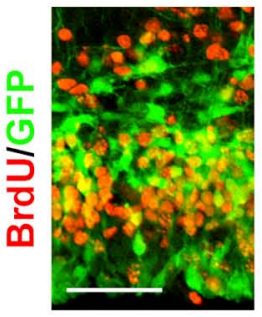

C

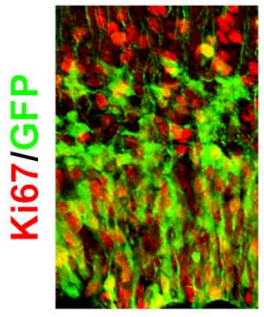

Pten protector
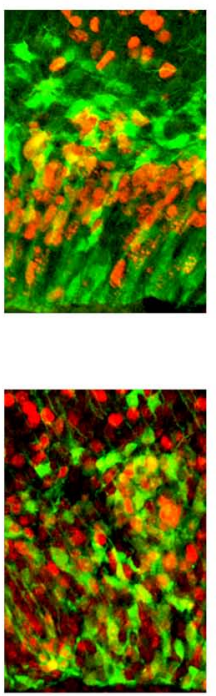
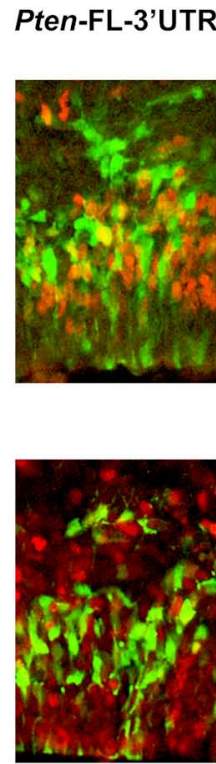

Pten-FL-3'UTR $+\operatorname{miR}-19 a$
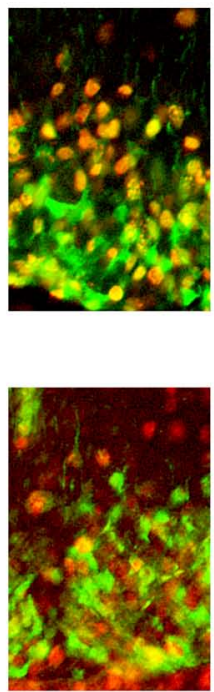

B

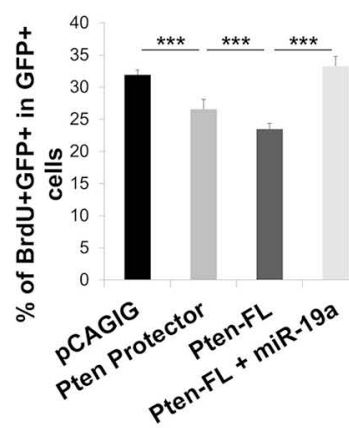

D

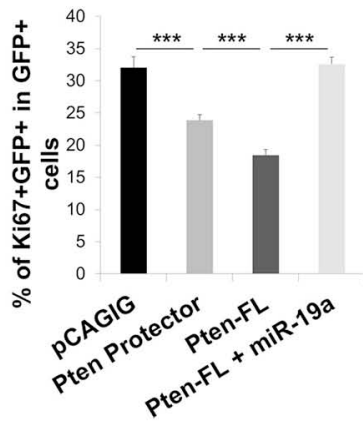

FIGURE 4 | Target protectors block miR-19a repression of Pten in the developing mouse cortex. (A-D) Electroporation of the $60 \mathrm{nt}$ target protector at E13.5 for analysis at E14.5 significantly decreased the number of $\mathrm{BrdU}$-incorporating or $\mathrm{Ki}^{+} 7^{+}$proliferative cells colabeled with GFP in the cortex. Ectopic expression of full length
Pten containing the $3^{\prime}$ UTR (Pten-FL-3'UTR) showed a similar effect, while co-electroporation of Pten-FL-3'UTR and miR-19a ablated this effect. Data are presented as mean $\pm \mathrm{SD} ; n \geq 3$ for all electroporations; $p$ values in relation to control $\left.{ }^{* * *} p<0.001\right)$. Scale bar: $50 \mu \mathrm{m}$.
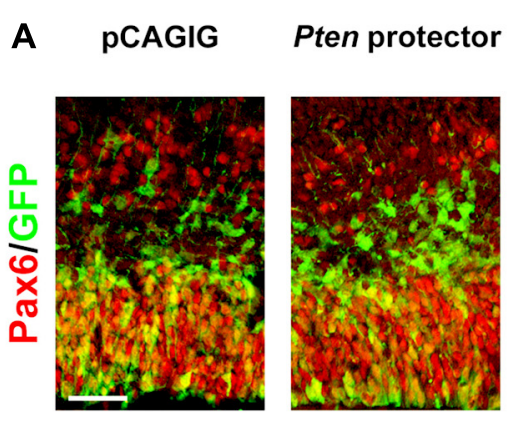

C

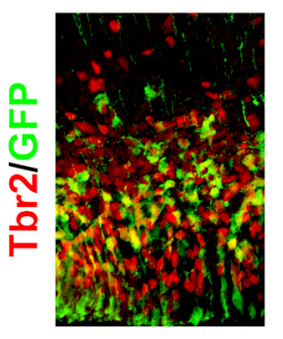

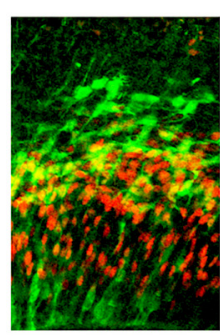
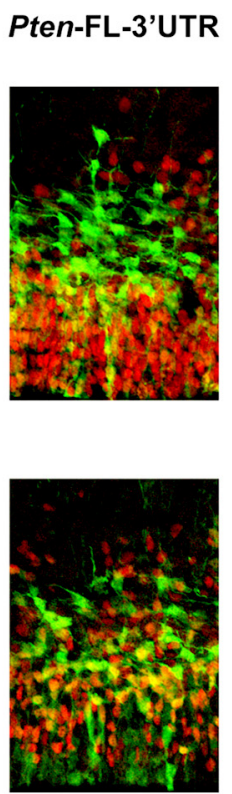
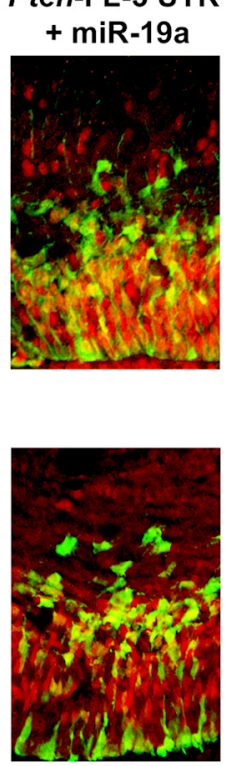

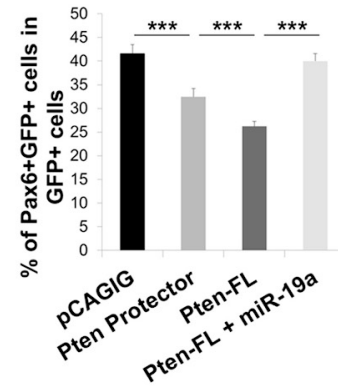

D

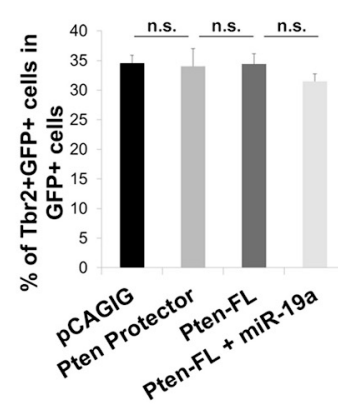

FIGURE 5 | miR-19a targeting of Pten is responsible for RGC expansion in the developing mouse cortex. (A,B) miR-19a targeting of Pten is important for RGC expansion. Introduction of the $60 \mathrm{nt}$ target protector at E13.5 for analysis at E14.5 significantly decreased the number of RGCs colabeled with GFP and Pax6. Ectopic expression of Pten-FL-3' UTR showed a similar effect, while co-electroporation of
Pten-FL-3' UTR and miR-19a ablated this effect. (C,D) Ectopic expression of the 60 nt target protector, Pten-FL-3'UTR, or Pten-FL-3'UTR and miR-19a had no effect on the number of IPs colabeled with GFP and Tbr2. Data are presented as mean $\pm \mathrm{SEM} ; n \geq 3$ for all electroporations; $p$ values in relation to control $(* * * p 0.001)$. n.s., not significant. Scale bar: $50 \mu \mathrm{m}$. 
in some model organisms, it is not applicable to many mammalian systems.

We have shown that plasmid-based target protectors are effective in mammalian systems. They are a simple and clean method for blocking miRNA action at a single target, eliminating uncertainty surrounding which mRNA targets are responsible for a phenotype. Because they are plasmid-based, these target protectors can be applied across a wide variety of model systems and tissues. They also have long lasting expression and can be used to study gene functions at any stage of development.

Here, we used in utero electroporation to introduce target protectors into the developing cortex; however, this is not the only application of plasmid-based target protectors. These protectors could also be used in viral transfection, or they could be incorporated to create transgenic lines. For any method of delivery, a conditional expression vector can be used to analyze a miRNA:target relationship in a specific cell type and at a specific stage. These target protectors also have disease treatment potential, as they could be used to increase expression of a dysregulated gene by blocking a miRNA.

While our plasmid-based target protectors are shown to be very effective for dissecting the miR-19a:Pten relationship, we have applied them to other miRNA:target pairs with mixed results. First, it is important to reiterate that these protectors are not applicable to every miRNA-binding site: if the protector overlaps another miRNA-binding site, protein binding site, or interferes with other RNA processing signals, then the results will be difficult to interpret. Another factor that may influence protector function is RNA secondary structure, either of the target RNA or the protector itself. To alleviate this problem, we recommend designing and testing target protectors of multiple lengths; though we found the

\section{REFERENCES}

Alvarez-Garcia, I., and Miska, E. A. (2005). MicroRNA functions in animal development and human disease. Development 132, 4653-4662. doi: 10.1242/dev.02073

Bartel, D. P. (2004). MicroRNAs: genomics, biogenesis, mechanism, and function. Cell 116, 281-297. doi: 10.1016/S0092-8674(04)00045-5

Bian, S., Hong, J., Li, Q., Schebelle, L., Pollock, A., Knauss, J. L., et al. (2013). MicroRNA cluster miR-17-92 regulates neural stem cell expansion and transition to intermediate progenitors in the developing mouse neocortex. Cell Rep. 3, 1398-1406. doi: 10.1016/j.celrep.2013.03.037

Bonev, B., Pisco, A., and Papalopulu, N. (2011). MicroRNA-9 reveals regional diversity of neural progenitors along the anterior-posterior axis. Dev. Cell 20, 19-32. doi: 10.1016/j.devcel.2010.11.018

Carrington, J. C., and Ambros, V. (2003). Role of microRNAs in plant and animal development. Science 301, 336-338. doi: 10.1126/science. 1085242
Choi, W.-Y., Giraldez, A. J., and Schier, A. F. (2007). Target protectors reveal dampening and balancing of nodal agonist and antagonist by miR-430. Science 318, 271-274. doi: 10.1126/science. 1147535

Conte, I., Carrella, S., Avellino, R., Karali, M., Marco-Ferreres, R., Bovolenta, P., et al. (2010). miR-204 is required for lens and retinal development via Meis2 targeting. Proc. Natl. Acad. Sci. U.S.A. 107, 15491-15496. doi: 10.1073/pnas.0914785107

Ebert, M. S., Neilson, J. R., and Sharp, P. A. (2007). MicroRNA sponges: competitive inhibitors of small RNAs in mammalian cells. Nat. Methods 4, 721-726. doi: 10.1038/ nmeth 1079

Ebert, M. S., and Sharp, P. A. (2010). MicroRNA sponges: progress and possibilities. RNA 16, 2043-2050. doi: 10.1261/rna.2414110

Friedman, R. C., Farh, K. K., Burge, C. B., and Bartel, D. P. (2009). Most mammalian mRNAs are conserved targets of microRNAs. Genome Res. 19, 92-105. doi: 10.1101/gr.082701.108

$60 \mathrm{nt}$ target protector to be the most effective, this may not be true in every case.

Another reason that a single target protector might not show a phenotype is that miRNAs often target multiple members of the same pathway. Thus, derepression of a single pathway member may be masked by continued regulation of the rest of the pathway. In this study, we successfully introduced target protectors for two miR-19a binding sites and we recommend this combinatorial approach for derepression of multiple pathway members. However, this method is limited by the maximum amount of DNA that can be introduced at a time. A large amount of each target protector is required to see an effect, which would be diluted by including multiple target protectors.

Plasmid-based target protectors open myriad opportunities in the miRNA field for dissecting specific miRNA:target interactions in mammalian model systems. These target protectors are applicable across tissues and developmental systems, and can be introduced in many ways. Here, we have presented basic concepts of target protector design and shown their application both in vitro and in vivo. We also revealed an important role of the miR19a:Pten interaction in the developing cortex, demonstrating the great potential of this essential new tool.

\section{ACKNOWLEDGMENTS}

We thank Andrew Pollock for helpful discussions of protector designs. This work was supported by the NIH T32 Training Grant in Developmental Biology at Weill Cornell Medical College (Jennifer L. Knauss), the Hirschl/Weill-Caulier Trust (Tao Sun), an NPRP grant (09-1011-3-260) from the Qatar National Research Fund and an R01-MH083680 grant from the NIH/NIMH (Tao Sun).

Groszer, M., Erickson, R., ScriptureAdams, D. D., Lesche, R., Trumpp, A., Zack, J. A., et al. (2001). Negative regulation of neural stem/progenitor cell proliferation by the pten tumor suppressor gene in vivo. Science 294, 2186-2189. doi: 10.1126/science. 1065518

Karres, J. S., Hilgers, V., Carrera, I., Treisman, J., and Cohen, S. M. (2007). The conserved microRNA miR-8 tunes atrophin levels to prevent neurodegeneration in Drosophila. Cell 131, 136-145. doi: 10.1016/j.cell.2007.09.020

Kloosterman, W. P., and Plasterk, R. H. (2006). The diverse functions of microRNAs in animal development and disease. Dev. Cell 11, 441-450. doi: 10.1016/j.devcel.2006.09.009

Landgraf, P., Rusu, M., Sheridan, R., Sewer, A., Iovino, N., Aravin, A., et al. (2007). A mammalian microRNA expression atlas based on small RNA library sequencing. Cell 129, 1401-1414. doi: 10.1016/j.cell.2007. 04.040

Lewis, B. P., Burge, C. B., and Bartel, D. P. (2005). Conserved seed pairing, often flanked by adenosines, indicates that thousands of human genes are MicroRNA targets. Cell 120, 15-20. doi: 10.1016/j.cell.2004.12.035

Lewis, B. P., Shih, I., Jones-Rhoades, M. W., Bartel, D. P., and Burge, C. B. (2003). Prediction of mammalian MicroRNA targets. Cell 115, 787-798. doi: 10.1016/S00928674(03)01018-3

Lim, L. P., Lau, N. C., Garrett-Engele, P., Grimson, A., Schelter, J. M., Castle, J., et al. (2005). Microarray analysis shows that some microRNAs downregulate large numbers of target mRNAs. Nature 433, 769-773. doi: 10.1038/nature03315

Loya, C. M., Lu, C. S., Vanvactor, D., and Fulga, T. A. (2009). Transgenic microRNA inhibition with spatiotemporal specificity in intact organisms. Nat. Methods 6, 807-903. doi: 10.1038/nmeth.1402

Mavrakis, K. J., Wolfe, A. L., Oricchio, E., Palomero, T., De Keersmaecker, K., McJunkin, K., et al. (2010). Genome-wide RNA-mediated interference screen identifies miR-19 targets in Notch-induced T-cell 
acute lymphoblastic leukaemia. Nat. Cell Biol. 12, 372-379. doi: 10.1038/ncb2037

Mu, P., Han, Y. C., Betel, D., Yao, E., Squatrito, M., Ogrodowski, P., et al. (2009). Genetic dissection of the miR-17-92 cluster of microRNAs in Myc-induced B-cell lymphomas. Genes Dev. 23, 2806-2811. doi: 10.1101/gad.1872909

Olive, V., Bennett, M. J., Walker, J. C., Ma, C., Jiang, I., CordonCardo, C., et al. (2009). miR-19 is a key oncogenic component of mir-1792. Genes Dev. 23, 2839-2849. doi: 10.1101/gad.1861409

Rasmussen, K. D., Simmini, S., AbreuGoodger, C., Bartonicek, N., Di Giacomo, M., Bilbao-Cortes, D., et al. (2010). The miR-144/451 locus is required for erythroid homeostasis. J. Exp. Med. 207, 1351-1358. doi: 10.1084/jem.20100458
Saito, T. (2006). In vivo electroporation in the embryonic mouse central nervous system. Nat. Protoc. 1, 1552-1558. doi: 10.1038/nprot.2006. 276

Sokol, N. S. (2005). Mesodermally expressed Drosophila microRNA-1 is regulated by Twist and is required in muscles during larval growth. Genes Dev. 19, 2343-2354. doi: 10.1101/gad.1356105

Stanton, A. A., and Giraldez, A. J. (2011). Use of target protector morpholinos to analyze the physiological roles of specific miRNAmRNA pairs in vivo. Nat. Protoc. 6, 2035-2049. doi: 10.1038/nprot.2011. 423

Ventura, A., Young, A. G., Winslow, M. M., Lintault, L., Meissner, A., Erkeland, S. J., et al. (2008). Targeted deletion reveals essential and overlapping functions of the miR-17-92 family of miRNA clusters. Cell 132, 875-886. doi: 10.1016/j.cell.2008. 02.019

Woltering, J. M., and Durston, A. J. (2008). MiR-10 represses HoxB1a and HoxB3a in zebrafish. PLoS ONE 3:e1396. doi: 10.1371/journal.pone.0001396

Zheng, H., Ying, H., Yan, H. Kimmelman, A. C., Hiller, D. J., Chen, A. J., etal. (2008). p53 and Pten control neural and glioma stem/progenitor cell renewal and differentiation. Nature 455, 1129-1133. doi: 10.1038/nature 07443

Conflict of Interest Statement: The authors declare that the research was conducted in the absence of any commercial or financial relationships that could be construed as a potential conflict of interest.
Received: 13 August 2013; accepted: 04 September 2013; published online: 24 September 2013.

Citation: Knauss JL, Bian S and Sun $T$ (2013) Plasmid-based target protectors allow specific blockade of miRNA silencing activity in mammalian developmental systems. Front. Cell. Neurosci. 7:163. doi: 10.3389/fncel.2013.00163

This article was submitted to the journal Frontiers in Cellular Neuroscience.

Copyright (C) 2013 Knauss, Bian and Sun. This is an open-access article distributed under the terms of the Creative Commons Attribution License (CC BY). The use, distribution or reproduction in other forums is permitted, provided the original author(s) or licensor are credited and that the original publication in this journal is cited, in accordance with accepted academic practice. No use, distribution or reproduction is permitted which does not comply with these terms. 